\title{
Analysis of mammogram using self-organizing neural networks based on spatial isomorphism
}

\author{
Aida A. Ferreira, Francisco Nascimento Jr., Ing Ren Tsang, George D. C. Cavalcanti, \\ Teresa B. Ludermir and Ronaldo R. B. de Aquino
}

\begin{abstract}
The correct segmentation and measurement of mammography images is of fundamental importance for the development of automatic or computer-aided cancer detection systems. In this paper we propose a method to segment mammogram image using a self-organizing neural network based on spatial isomorphism. The method used is a modified version of the algorithm proposed by Venkatesh and Rishikesh [1] to extract object boundaries in an image. This model explores the principle of spatial isomorphism and selforganization in order to create flexible contours that characterize shapes in images. We modified the original algorithm to overcame problems of local minimum, poor performance for image object with large concavity and imprecise results when simple or far from object border contour are chosen. A comparison of both algorithm and original segmentation used by the MIAS database [9] is presented.
\end{abstract}

\section{INTRODUCTION}

$\mathrm{T}_{\mathrm{t}}$ HE main objective of this paper is to develop a method to segment objects (tumors) in a mammogram image. Presently there has been an enormous interest in image processing and analysis techniques in mammography [14][15][16], since breast cancer is the leading type of cancer in women and the second most fatal type of cancer [17]. Early detection is of most importance for survival, but manual diagnosis is tedious, time consuming, and requires an expert [14][15]. Mammograms are the best method of detecting breast cancer; its results can indicate cancer years before physical symptoms occur. Doctors may choose to use an automated detection system to help or simply as an aid in locating suspicious masses [15]. The precise localization and correct segmentation and measurement of the image tumor are of importance. Here, we propose a method to segment mammogram image using a self-organizing neural network based on spatial isomorphism.

Manuscript received January 31, 2007. This work was supported in part by the Brazilian National Research Council CNPq (Proc. 478534/2006-0).

Authors are with Center of Informatics (CIn), Federal University of Pernambuco (UFPE), P.O. Box 7851, Cidade Universitária, Cep: 50.740530 - Recife - PE - Brazil (corresponding authors to provide phone: 55-8121268430;e-mails: aaf@cin.ufpe.br; fnj@cin.ufpe.br; tir@cin.ufpe.br; gdcc@cin.ufpe.br, tbl@cin.ufpe.br).

Ronaldo R. B. de Aquino, is with the Federal University of Pernambuco (UFPE), Academico Helio Ramos s/n, Cidade Universitária, Cep: 50.740530 - Recife - PE - Brazil (corresponding author to provide phone: 55-8121268986; e-mail: rrba@ufpe.br).
There are many techniques based in models, which uses an initial contour that adjusts to the border of the object of interest. The models used in these processes can be rigid or flexible, such as active contour models (ACM) or deformable templates. An ACM algorithm utilizes an initial contour defined as the border object that modifies itself until the contour approximates the border of the object. The word active is used to represent the dynamic nature of the model in the process to define the final contour. Due to the flexibility of active contour models, they have been used to solve various problems in computer vision, such as: stereovision, movement tracking, contour detection and face recognition [1][7][8].

The problem discussed in the paper is the localization and extraction of the tumor region (salient contour) in the mammogram images. We use an ACM based on selforganizing network (SON)[4][5] to segment the region of interest in the image. This model explores the principle of isomorphism and self-organization to create flexible contours that characterizes the shapes in the image [1][7][8].

The flexibility of the model is obtained by a scheme of local cooperation and global competition in the selforganizing neural networks, which allows the inclusion of the nearest salient contour of the image. This model requires a rough border as initial contour to start the process of deformation. Also, the technique is semi-automatic since it needs an initial contour. However, it is useful in various different types of problems such as border extraction, medical images and digital libraries [1][2][3][7][8].

The model proposed by Venkatesh and Rishikesh [1] is completely different from other models of active contour in both theory and implementation. They used a modified neural networks proposed by Ganesh Murthy and Venkatesh [2] and used by Shanmukh et al [3], that applies isomorphic self-organizing networks (SON) to the patterns. Venkatesh and Rishikesh explored the simplicity and elegance of this model to adapt to the problem of contour extraction. However, this method presents the same difficulties found in other ACM, which are problems in local minimum, poor modeling for object with large concavity and imprecise results, if a simple or far away initial border is used. We propose a modified version of this model that minimizes these problems. 


\section{ACTIVE CONTOUR MODELS}

\section{A. Definition}

The first and most important step in image analysis is the segmentation procedure (generally referred as border estimation, edge detection or object localization). Segmentation subdivides an image in different basic constituent parts. Segmentation algorithms for gray level images are generally based on two fundamental properties: discontinuity and similarity. The first method divides the image based on sudden changes in the gray levels (isolate points, lines and borders), while the second method is based on threshold, growth region and division and fusion of regions.

Both methods use information present only in the image. However the problem with these procedures is that the border obtained might not be the actual object borders. Except images of very high quality obtained in controlled environments these methods can create false border and wholes. In general, they are also limited and useless in images of low quality. The limitations of these methods are due to the use of information only on the neighborhood of the local image. They ignore information based on image model and border grouping. These methods are considered low-level mechanisms [6][7][8]. Alternatively we can use high-level segmentation methods that utilize prior knowledge of the shape, texture, color or object position in the segmentation procedure.

Active Contour Models (ACM) or snakes are curves generated by computer that moves along the image in search of the border of the object [6]. They are often used in computer vision and image analysis to localize objects and to describe its shapes. For example, a snake can be used to find a region in a medical image or to identify a character in a handwritten letter. They are defined as partial differential equations and are considered an ACM method. Snakes can be implemented using an implicit finite difference or through dynamical programming or using methods of finite elements. The first two methods are more common in the literature [7][8]. Some applications of object segmentation using ACM are: snakes, balloons, template deformation, and dynamical contours.

\section{B. Snakes}

Snake is a parametric curve defined in the image domain. All the proprieties of Snake and its behavior are specified through an energy function in analog with a physical system. A partial differential equation controlling a Snake has the objective to minimize the energy; the analogy with a physical system can be extended to the notion of Snake [13]. Let's image that a border map is a barrier, in which the parametric curve can be moved, force acting upon the curve moves the barrier trying to restore the energy equilibrium.
The model that moves the barrier has two components: one that controls the behavior (to preserver the original form, to develop a curve, etc) the other controls the direction. The objective is to the curve adjusts itself to the border of the specific object in the image. The border can be recognized as the low values of the negative border map; therefore the equilibrium equation should minimize the term involved in the negative border or the potential energy of the dynamical system. Since the general form of the object is known, we can draw the evolution equation in which a Snake can easily hold the object, which should be elastic, firm and capable to develop a curve or something similar. Kass, Witkin and Terzopoulous proposed the first Snake model in 1987 [6].

\section{Deformation template}

Deformation template is another technique to approximate objects. This method applies a prior knowledge of the shape of the object, which is obtained through a draft, binary template or parametric prototype. The prior information is coded in the shape of the binary border in a template or parameter vector. The information does not need to be extract in a sense that it does not need to correspond exactly to the borders of the image [11].

The difference between Snakes and deformation template is that Snake are functions that minimize free energy of the shape. In the Snake model there are no global structure in the curve except for some restriction that regulates the smoothness and continuity of the border. On the other hand, deformation templates control the deformation using a set of parameters capable to codify a specific shape. This type of model is used in case that more specific information about the shape, that can be described in a binary template or in a set of parameters, is available.

\section{Dynamic Contour}

Both Snakes and deformation templates can be classified as dynamical contours, since they utilize a dynamic environment [12]. ACM can be applied to simple static images or to a sequence of dynamical images. In dynamical applications some additional moments can be incorporated in the model, so that any prior knowledge about the object and deformation can be expressed. In contrast, using Snakes only the active contour varies, the dynamical contour of the border map is also variable. Snake can also be applied in a sequence of images. This group of ACM has application in traffic monitoring, visual speech recognition, movement control and others. ACM can be classified according to some difference criteria; ACM free of form and ACM with limited form.

There are no global structures for the contours in the free of form ACM. The limitation is due to local continuity restrictions and smoothness. Also, there is no use of prior 
information upon the form since this information is used to adjust the model parameters over the contour proprieties (elasticity, rigidity, etc) that enable the procedure to encircle the object of interest.

The limited form ACM uses prior information on the direction of the geometrical shape. This information is available as a vector of parameters, which codes the shape of interest. The geometrical shape of the contour is adjusted through the variation of the parameters. Deformation templates are example of limited form ACM. The reason to call them limited is because they cannot take any arbitrary shape. Shape validity is limited through the prototype of the template.

ACM can also be classified by the information obtained in the shape of the image used to align the contour with the object of interest. The model can be classified as: region based model or border-based model. Region based models represents a contour of the image segment within welldefined region. The image is examined to decide if a pixel belongs to the object or if it is outside the frontier of the object. A pixel belongs to the border if it is in the region of the object and its neighbors are in the background. Segmentation is used to produce an image with a force field that aligns the active contour with the object of interest.

\section{ISOMORPHISM}

Based on the recognition of the human vision system, the use of spatial isomorphism for object and character recognition was identified as a strategy to maintain the characteristics of the human vision system, like scale changes, rotation and shift. For this reason, in the implementation of the model, we used a self-organization scheme similar to the model of Kohonen, but different in terms of architecture, since it was not used a lattice of neurons.

\section{MODELING ACTIVE CONTOUR THROUGH SON NETWORK}

Venkatesh and N. Rishikesh [1] introduced Selforganizing Network (SON) by as an alternative for the traditional active contour model (ACM)[6]. SON network is adapted to solve the problem of border extraction of objects in an image, which is done by minimization of functions based on energy. The network, that has a fixed number of neurons in a ring topology, is created in an isomorphic way for an initial condition (one-to-one correspondent between the contour points and the networks points), initializing a contour and being deformed until the image contour is mapped. Figure 1(a) shows a traditional SON network topology and figure 1(b) shows the topology of a SON network based on ACMs.

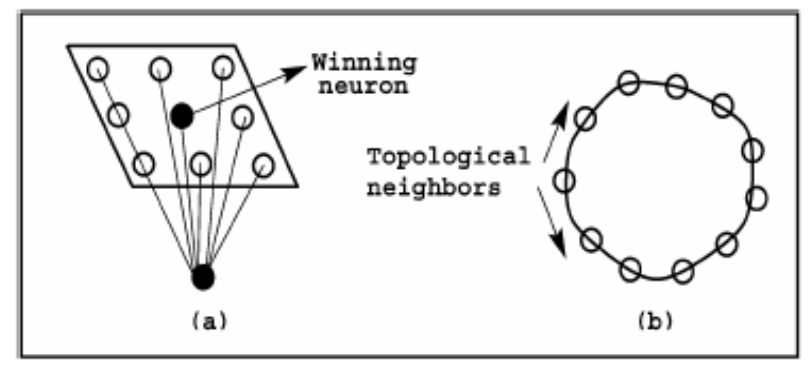

Fig 1. (a) Lattice topology employed in conventional Kohonen-SOM algorithms. (b) Chain topology used in SOM-based ACMs.

The primary limitations of SON-based ACMs are the condition of the initial contour, which must be: near the border of the object and similar to the shape of the object.

This is due to the fact that the neighborhood function in the update of the weights is based only on the physical distance between the neurons and not on the indices. In this way the update of the weights turn gradually smaller when the neurons are distant of the winner, even if they are topologically neighbors. As long as the number of contour points remains fixed, the final contour will not be a precise representation of the real border. If the contour is not specified by a set of continuous points, the algorithm fails.

The model proposed by Venkatesh and Rishikesh [1] produces good results when the initial contour is near the border of the object. Even then it might happen that some parts of the initial contour never gets near the borders, mainly when the initial contour is far away of the object or when the object have concavities. We proposed a modified algorithm that is able to minimize these problems.

\section{PROPOSED ACTIVE CONTOUR MODEL}

With the objective of minimizing the problems of initial contour far from the object border and object concavity, we implemented the algorithm proposed in [1] with a penalty to the winner neurons in the next interactions. Like most of the ACM models, our proposed model needs an initial contour that involves the object of interest. The initial contour used in the test is the circle indicated in each of the images [9]. A neural network isomorphic to the initial contour is build, which is followed by a procedure that modifies this contour with the objective of obtaining the most accurate contour of the object in the image. The steps involved in this procedure are the following:

1. Apply an image filter to smooth eventual noise, which appears from the image acquisition procedure. We used a median filter with window size varying from 3 to 9 pixels.

2. Normalize the image to enhance the desired object 
(tumor). We divided the gray levels of the images in 64 bands. Since the objects (tumors) that we want to localize have a higher gray level value, we changed the $f$ greater gray levels bands in a value equal 255 . The parameter $f$ varies from 8 to 20 .

3. Compute the edge map of the image, because the method needs a rough boundary as the initial contour to start with the deformation process. We used a Sobel method instead of the Hough transform originally used in [1] because of its excessive requirement for memory and computation time especially as the number of parameters increases [10].

4. Obtain the edge points $E=\left\{\left(x_{i}, y_{i}\right), i=1, \ldots, N_{e}\right\}$ within the region of interest, where $N_{e}$ is the number of edge points within the region of interest.

5. Construct a network with $N_{c}$ neurons, where $N_{c}$ is the number of points on the initial contour. Each neuron in the network receives three inputs $\left(I_{1}, I_{2}, I_{3}\right)$. The weights $w^{i}=\left(w_{1}^{i}, w_{2}^{i}, w_{3}^{i}\right), i=1, \ldots, N_{c}$, corresponding to these three inputs, are initialized to the co-ordinates of points on the initial contour $\left(w_{1}^{i}, w_{2}^{i}\right)$ and the penalty equals zero $\left(w_{3}^{i}\right)$. The penalty weight $\left(w_{3}^{i}\right)$ is useful to avoid that the same neuron becomes the winner repeatedly.

6. Repeat the following steps a certain number of times $\left(N_{\text {iter }}\right)$ :

(i) Select a point $\mathrm{p}=(u, v) \in E$ randomly, and feed the $(x, y)$ co-ordinates of the selected point $\mathrm{p}$ as inputs $\left(I_{1}, I_{2}\right)$ to every neuron in the network.

(ii) Determine the neuron whose weight vector is closest to the input vector, and declare it as the winner neuron. Compute the distance using

distance $=$ Euclidian distance measure $+w_{3}^{i}$

The penalty weight $\left(w_{3}^{i}\right)$ is used to give a chance to another neuron to be the winner, since each time that a neuron is the winner this parameter is increased. If the distance between the vector $\left(W^{w}\right)$ and the input vector is greater than a particular threshold $T_{w d}$, then go to (i).

(iii) Add the penalty parameter ( $\alpha$ ) to winner neuron's weight $w_{3}^{i}$. (iv) Update the weights of the neurons in the network using the following rule:

For neuron $i$,

$w^{i}=w^{i}+\eta^{*} \exp \left(\frac{-\left\|w^{w}-w^{i}\right\|^{2}}{2 \sigma^{2}}\right) *\left(p-w^{i}\right)$

where $\eta, \sigma$ are the standard learning rate and neighborhood parameters.

(v) Calculate the parameter $C_{n p}$ (neighborhood parameter) of the contour as:

$C_{n p}=\operatorname{Max}\left\{\operatorname{Max}\left(\left|w_{1}^{i}-w_{1}^{i+1}\right|,\left|w_{2}^{i}-w_{2}^{i+1}\right|\right): 1 \leq i \leq N_{c}-1\right\}$

If $C_{n p}>T_{n p}$ the threshold value of the neighborhood constraint parameter, then restore the previous network weights discarding the present update.

Vary $\eta$ and $\sigma$ according to the following rules:

$$
\begin{gathered}
\eta=\eta_{\text {init }} *\left(\eta_{\text {fin }} / \eta_{\text {init }}\right)^{i t e r / N_{\text {iter }}}, \\
\sigma=\sigma_{\text {init }} *\left(\sigma_{\text {fin }} / \sigma_{\text {init }}\right)^{i t e r / N_{\text {iter }}},
\end{gathered}
$$

where $\eta_{\text {ini and }} \eta_{\text {fin }}$ initial and final values of $\eta ; \sigma_{\text {ini }}$ and $\sigma_{\text {fin }}$ are those of $\sigma$; and iter is the current iteration number.

The algorithm was implemented using Matlab Version 7 R13. The images used in this work were obtained from MIAS MiniMammographic Database. By popular request the original MIAS Database (digitized at 50 micron pixel edge) has been reduced to 200-micron pixel edge and clipped/padded so that every image is 1024 pixels x 1024 pixels. The images are available in [9].

\section{RESULTS}

We tested the proposed modifications of the original algorithm using the images taken from the MIAS database of mammograms [9]. The original image shows a circular object that represents the tumor as shown in the figures.

Since the algorithm needs a rough initial contour to start the process of deformation, we used the circle defined by the images of the MIAS database as initial contour. The encircled region indicates the location of a suspected tumor. Because of the isomorphism, the size of the net (number of neurons) depends in the initial selected contour. If the initial 
contour has $n$ pixels the size of the net will consequently also be $n$.

The constraint on the winner-distance ( $T_{w d}$ parameter) is useful in avoiding the influence of edge points, which are within the region of interest, but are not a part of the nearest salient contour of interest [1]. In the absence of such a constraint, the neurons organize themselves to spurious edges. If the distance between the input vector and the winner neuron's vector weight is greater than $T_{w d}$, then the weights of network are not update.

The neighborhood constraint ( $T_{n p}$ parameter $)$ refers to maximum of the distances in $x$ - and $y$-directions, taken over of all the adjacent pairs of points on the contour. Constraining this parameter helps in maintaining the continuity of the contour in the course in the course of its deformation [1]. In the absence of this parameter many neurons tend to organize themselves towards a single point of the input image, leading to discontinuities in the final contour.

The penalty parameter $(\alpha)$ is utilized in avoiding the same neuron became winner repeatedly, because each time that a neuron is a winner the weight $\left(w_{3}^{i}\right)$ is increased by $\alpha$. Using this parameter it was possible to overcome problems of local minimum, poor performance for image object with large concavity and imprecise results when simple or far from object border contour is chosen.

TABLE I

PARAMETERS

\begin{tabular}{lcccccccc}
\hline \hline File & $N_{\text {iter }}$ & $\eta_{\text {ini }}$ & $\eta_{\text {fin }}$ & $\sigma_{\text {ini }}$ & $\sigma_{\text {fin }}$ & $T_{w d}$ & $T_{n p}$ & $\alpha$ \\
\hline mdb001 & 2000 & 0.90 & 0.01 & 5 & 0.10 & 132 & 132 & $\mathbf{2 5}$ \\
$\operatorname{mdb010}$ & 700 & 0.70 & 0.01 & 5 & 0.20 & 26 & 26 & $\mathbf{5}$ \\
$\operatorname{mdb} 015$ & 1200 & 0.70 & 0.01 & 5 & 0.20 & 61 & 61 & $\mathbf{1 5}$ \\
$\operatorname{mdb} 021$ & 1200 & 0.70 & 0.01 & 5 & 0.20 & 44 & 44 & $\mathbf{1 5}$ \\
$\operatorname{mdb} 023$ & 700 & 0.70 & 0.01 & 5 & 0.20 & 17 & 17 & $\mathbf{1 0}$ \\
$\operatorname{mdb} 025$ & 1200 & 0.70 & 0.01 & 5 & 0.20 & 76 & 76 & $\mathbf{2 0}$ \\
$\operatorname{mdb} 028$ & 1200 & 0.70 & 0.01 & 5 & 0.20 & 50 & 50 & $\mathbf{1 5}$ \\
$\operatorname{mdb} 069$ & 1000 & 0.70 & 0.00 & 3 & 0.10 & 40 & 40 & $\mathbf{2 0}$ \\
$\operatorname{mdb} 091$ & 1200 & 0.70 & 0.01 & 5 & 0.20 & 14 & 14 & $\mathbf{1 0}$ \\
$\operatorname{mdb} 132$ & 1200 & 0.70 & 0.01 & 5 & 0.20 & 42 & 42 & $\mathbf{2 0}$ \\
\hline \hline
\end{tabular}

Table I shows the parameters used to test the algorithms using the images selected from the database (the first column shows the file name of these images).

\begin{tabular}{crrrr}
\hline \hline File & $\begin{array}{c}\text { Original } \\
\text { circle }\end{array}$ & $\begin{array}{c}\text { Original } \\
\text { algorithm }\end{array}$ & $\begin{array}{c}\text { Modified } \\
\text { algorithm }\end{array}$ & $\begin{array}{c}\text { Difference } \\
\text { between } \\
\text { algorithms (\%) }\end{array}$ \\
\hline mdb001 & 67853 & 58979 & 30167 & -48.85 \\
mdb010 & 3405.5 & 2794 & 2726 & -2.43 \\
mdb015 & 14499 & 12706 & 10464.5 & -17.64 \\
mdb021 & 7526.5 & 6294 & 4820 & -23.42 \\
mdb023 & 2628 & 2559.5 & 2475.5 & -3.28 \\
mdb025 & 22136 & 18426 & 10578 & -42.59 \\
mdb028 & 9822 & 7582 & 6459.5 & -14.80 \\
mdb069 & 6067.5 & 5455.5 & 2176 & -60.11 \\
mdb091 & 1246.5 & 1227.5 & 1171.5 & -4.56 \\
mdb132 & 6921.5 & 5506 & 1711.5 & -68.92 \\
\hline \hline
\end{tabular}

Table II, shows the total area (values in pixel count) of the encircled image indicating in [9], the obtained area for the original algorithm [1] and the area obtained with our proposed modified. These values represent the size of the suspected region, allowing a more accurate measurement of the tumor region. In the last column, it is shown the difference of area, in percentage, comparing both algorithms.

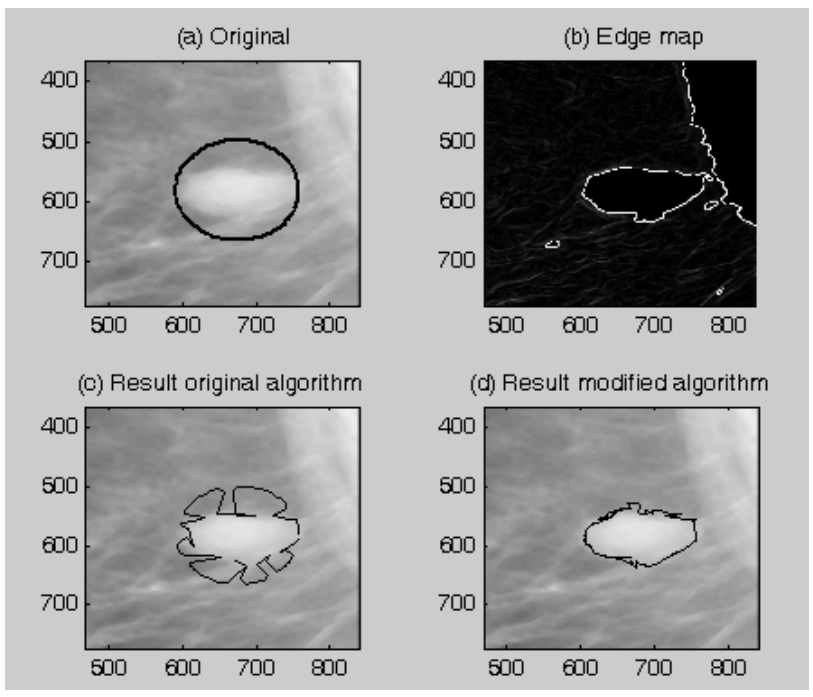

Fig. 2 (a) Original image mdb0025. (b) Edge map. (c) Final contour of the original method (d) Final contour of the modified algorithm.

Figure 2 shows the segmented images of the original database, the edge map used, the resulting segmentation using Venkatesh and Rishikesh algorithm and the segmented image obtained by our modified algorithm. Figures 2 to 4 correspond to the processed images mdb0025, mdb0028 and mdb0021, respectively. 


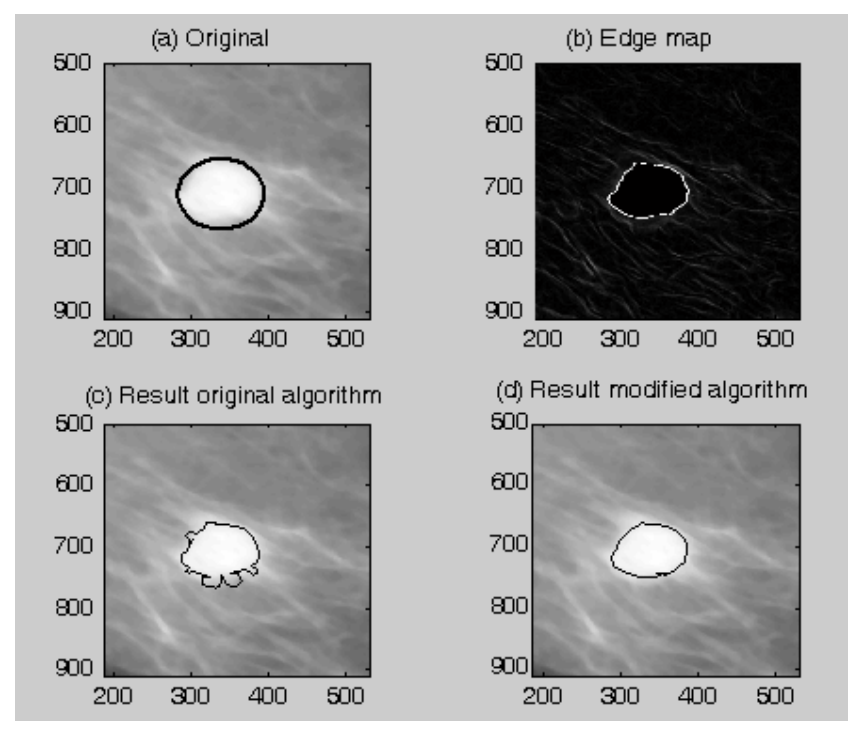

Fig. 3 (a) Original image. (b) Edge map. (c) Final contour of the original. method (d) Final contour of the modified algorithm.

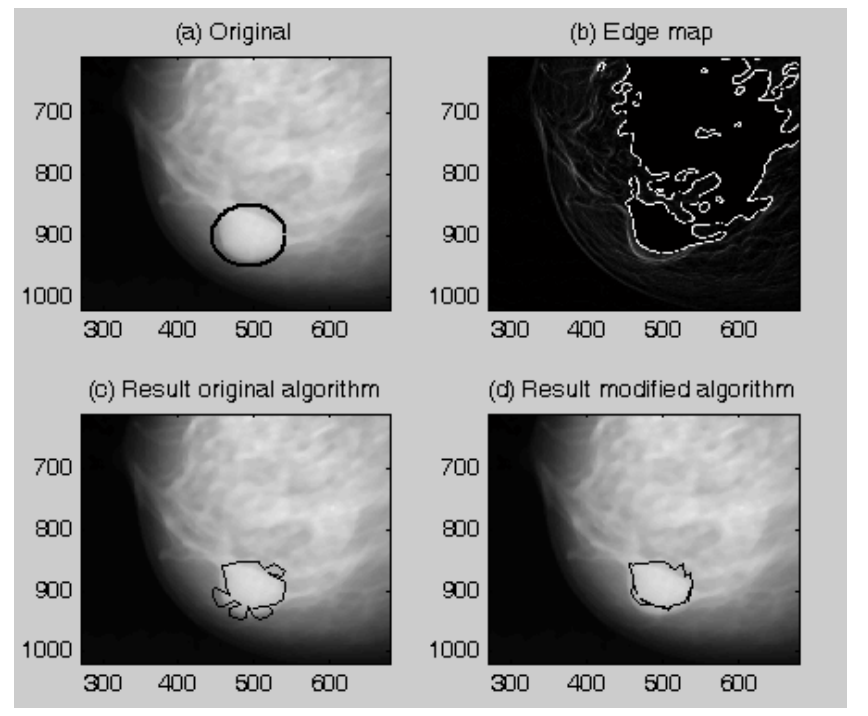

Fig. 4. (a) Original image. (b) Edge map. (c) Final contour of the original. method (d) Final contour of the modified algorithm.

In all the images tested, we confirm the problem that the active contour model have in dealing image objects with large concavity and distant from the object border. In Figures 2(c), 3(c) and 4(c), we observe the formation "bubble" areas using the original algorithm. The (d) images in these figures show the results for the modified algorithm, the segmented region represents a closer approximation of the tumor region.

\section{CONCLUSIONS}

We have shown the exploration of the isomorphism and self-organization principles applied to create flexible contours to enhance shapes of the images. The main contribution of this model is segmentation procedure, which performs a more accurate tumor region selection in mammogram images. We believe that the improved algorithm will also perform better than the original in other types of image segmentation problem since it handles better the contour minimization process.

The principal advantage of this modified algorithm is that it avoids the traditional problems of the ACM models with distant border contour and objects with large concavity.

\section{REFERENCES}

[1] Y. V. Venkatesh and N. Rishikesh. "Self-organizing neural networks based on spatial isomorphism for active contour modeling" in Pattern Recognition. vol. 33. J. Peters. Ed. Elsevier. 2000. pp. 1239-1250.

[2] N.S. Ganesh Murthy. Y.V.Venkatesh. "Pattern encoding and classification by neural networks". Neural Computing submitted for publication.

[3] K.Shanmukh. C.N.S. Ganesh Murthy. Y.V.Venkatesh. "Classification using self-organizing networks spatially isomorphic to patterns". International Conference on Robotic Vision for Industry and Automation. ROVPIA96. Malaysia. 1996. pp. 298-303.

[4] A.P.Braga. T.B.Ludermir. A.C.P.L.F.Carvalho. Redes Neurais Artificiais - Teoria e Aplicações. Editora LTC. 2000.

[5] S. Haykin. "Neural Networks: a comprehensive foundation". Prentice Hall, Inc. 1999.

[6] M.Kass. A.Witkin. D.Terzopoulos. Snakes: Active Contour Models. International Journal of Computer Vision. 1988. pp321-331.

[7] H.Shah-Hosseini. R.Safabakhsh. A TASOM-based algorithm for active contour modeling. Pattern Recognition Letters. 2002. pp. 13611373

[8] Y.V.Venkatesh. S.K.Raja. N.Ramya. Multiple Countor Extraction Graylevel Images Using an Artificial Neural Networks. IEEE Transactions on Image Processing. 2006. vol. 15. n. 4. pp. 1057-7149.

[9] http://peipa.essex.ac.uk/info/mias.html. "mini-MIAS database of mammograms".

[10] V. Hadziavdic. "A Comparative Study of Active Contour Models for Boundary Detection in Brain Images". Diploma Project. Faculty for Mathematical and Natural Sciences, University of Tromso, 1999.

[11] D. Terzopoulous and K. Fleischer, "Deformable models", Vis. Comput. Vol. 4, pp. 306-331, 1988

[12] R. Durikovic, K. Kaneda, and H. Yamashita, "Dynamic contour: A texture approach and contour operations," Vis. Comput. Vol 11, pp. 277-289, 1995.

[13] Chenyang Xu, Jerry L. Prince, "Snakes, Shapes, and Gradient Vector Flow", IEEE Transaction on Image Processing, vol. 7, no. 3, pp. 359$369,1998$.

[14] Isaac N. Bankman, Tanya Nizialek, Inpakala Simon, Olga B. Gatewood, Irving N. Weinberg and William R. Brody, "Segmentation Algorithm for Detecting Microcalcifications in Mammograms," IEEE Transaction on Information Technology in Biomedicine, vol. 1, no.2, pp. 141-149, 1997.

[15] H. D. Cheng, Xiaopeng Cai, Xiaowei Chen, Liming Hu, Xueling Lou, "Computer-aided detection and classification of microcalcifications in mammograms: a survey," Pattern Recognition 36, pp. 2967-2991, 2003

[16] C. B. R. Ferreira, D. L. Borges, "Analysis of mammogram classification using a wavelet transform decomposition," Pattern Recognition Letters 24, pp. 973-982, 2003.

[17] A.Jebal, T. Murray, A. Samuels, A. Ghafoor, E. Ward, M. Thun, "Cancer statistics, 2003," CA Cancer J. Clin., vol. 53, pp.5-26, 2003. 